\title{
The Unexplored Role of Handshape Similarity in Processing Numbers on the Hands
}

\author{
Ilaria Berteletti ${ }^{1}$ (1) , SaraBeth J. Sullivan ${ }^{1}$ (1) , Lucas Lancaster ${ }^{2}$ (1) \\ [1] PhD in Educational Neuroscience Program, Gallaudet University, Washington, DC, USA. [2] Hearing and Speech Center, School of Human Services and Sciences, \\ Gallaudet University, Washington, DC, USA.
}

Journal of Numerical Cognition, 2021, Vol. 7(2), 156-171, https://doi.org/10.5964/jnc.6997

Received: 2021-01-10 • Accepted: 2021-06-16 • Published (VoR): 2021-07-23

Handling Editor: Joonkoo Park, University of Massachusetts Amherst, Amherst, MA, USA

Corresponding Author: Ilaria Berteletti, 800 Florida Ave NE, Washington, DC 20002, USA. E-mail: ilaria.berteletti@gallaudet.edu

\begin{abstract}
With two simple experiments we investigate the overlooked influence of handshape similarity for processing numerical information conveyed on the hands. In most finger-counting sequences there is a tight relationship between the number of fingers raised and the numerical value represented. This creates a possible confound where numbers closer to each other are also represented by handshapes that are more similar. By using the American Sign Language (ASL) number signs we are able to dissociate between the two variables orthogonally. First, we test the effect of handshape similarity in a same/different judgment task in a group of hearing non-signers and then test the interference of handshape in a number judgment task in a group of native ASL signers. Our results show an effect of handshape similarity and its interaction with numerical value even in the group of native signers for whom these handshapes are linguistic symbols and not a learning tool for acquiring numerical concepts. Because prior studies have never considered handshape similarity, these results open new directions for understanding the relationship between finger-based counting, internal hand representations and numerical proficiency.
\end{abstract}

\section{Keywords}

numerical distance effect, number handshapes, handshape similarity, ASL number signs, ASL number comparison

Numerous studies appear to show that fingers might be related to numerical and arithmetical proficiency in more than one way, both in children and in adults (Berteletti \& Booth, 2016, for a comprehensive review). Gerstmann (1940), in the first half of the twentieth century, identified a constellation of deficits as the results of a lesion near the angular gyrus that included among others, acalculia and the inability to recognize and distinguish one's fingers (i.e., finger agnosia). Concurrently, Strauss and Werner (1938) were the first to find a relationship between finger gnosis and arithmetical skill in a group of children with low math achievement but normal IQ. However, it is only at the turn of the twenty-first century that renewed interest emerged on the relationship between finger representations and numerical performance.

More recently, different studies have investigated how finger-based strategies and internalized finger representations may relate to arithmetic proficiency but also more broadly to numerical and quantity processing. The relationship between finger gnosia and proficiency in arithmetic in children appears to show mixed results where some have found that finger gnosis correlates and selectively predicts later numerical and arithmetic ability in children (Fayol, Barrouillet, \& Marinthe, 1998; Newman, 2016; Noël, 2005; Reeve \& Humberstone, 2011) whereas others have failed to find such relationship (Long et al., 2016; Malone, Burgoyne, \& Hulme, 2020). It remains that studies with adults have shown how the internal representation of the finger-based counting sequence influences Arabic digit comparison and arithmetical calculations (Di Luca, Granà, Semenza, Seron, \& Pesenti, 2006; Domahs, Krinzinger, \& Willmes, 2008; 
Domahs, Moeller, Huber, Willmes, \& Nuerk, 2010; Fischer, 2008). For example, Domahs and colleagues (2010) compared groups of participants that either used one or two hands to count items up to 10 (i.e., German and German sign language vs Chinese counting systems) on an Arabic digit comparison task. The authors found that comparing Arabic digits across the boundary of 5 was slower for the groups using the 2 hands counting system and interpreted the results as indicative of an internalized sub-base-five representation driven by an internalized representation of the hand configurations for counting. The specific configurations of how individuals count on their fingers has also been shown to be associated with numerical representations (Badets \& Pesenti, 2010; Badets, Pesenti, \& Olivier, 2010; Di Luca et al., 2006; Di Luca \& Pesenti, 2008). In one study, Di Luca and colleagues (2006) tested if individual fingers could be associated with specific numerical magnitudes due to internalized finger-based counting procedures. Using an Arabic digit-to finger mapping design, they found that participants were faster at identifying 1 of 10 keys if the numerical mapping of the keys corresponded with the participant's canonical finger-counting habit, that is with how they would sequentially raise their fingers when counting up to 10 . These results were interpreted as automatic associations between the personal handshapes for counting and the numerical representations as a consequence of the repeated use of such sequence either when learning how to count or as a working memory relief strategy for calculating. Further, the same authors found that presenting hand configurations that matched with personal hand counting sequences, also defined as canonical configurations, not only primed the processing of the subsequent Arabic digit faster than non-canonical configurations, but they did this in a qualitatively different way suggesting a preferential link with the internal numerical representation (Di Luca, Lefèvre, \& Pesenti, 2010; Di Luca \& Pesenti, 2008). The preference for canonical configuration over non-canonical configurations has also been found in children as early as in kindergarten (Lafay, Thevenot, Castel, \& Fayol, 2013; Nicoladis, Marentette, Pika, \& Barbosa, 2018). These results suggest that handshapes for representing numbers might start to be internalized early when learning about numbers. Indeed, a neuroimaging study with preadolescents found that the motor and somatosensory representations of the fingers were activated while solving single-digit operations and this activation was modulated by operation type, problem size and performance even in the absence of movement (Berteletti \& Booth, 2015).

Although some studies have questioned whether the use of fingers counting and finger-based strategies is necessary or simply a useful tool (Crollen, Seron, \& Noël, 2011; Lafay et al., 2013), the different lines of research still suggest that finger-based strategies used during childhood may play a functional role in supporting numerical representations and calculation (Andres \& Pesenti, 2015; Berteletti \& Booth, 2016; Di Luca \& Pesenti, 2011; Fischer \& Brugger, 2011; Soylu, Lester, \& Newman, 2018).

Interestingly, on the one hand, studies have debated on whether the somatosensory representation of one's fingers is related to arithmetic performance in children; on the other, studies have shown that processing numerical information presented as Arabic digits is influenced by personal representations of finger configurations. However, no study has directly investigated whether the hand configuration itself could induce its own effect and influence the processing of numerical information. In most cultures (Bender \& Beller, 2012, for a review), there is a relationship between the number of fingers raised (or folded) and the number represented, at least for numbers up to five. Thus, comparing the representations of 2 and 5 presented on the hands involves not only accessing the numerical representation but also distinguishing between the representations of handshapes with two versus five fingers. When observing the hand configurations, it also appears that the handshapes presenting numerically closer values also share more features and appear more similar. The question therefore is whether the hand configuration itself, with shared raised or bent fingers, can interfere with the actual numerical processing.

The overall aim of this study is to test the hypothesis that recognizing hand configurations might present its own, distinct processing effects and that it might interfere when processing numerical information presented on the fingers. In two experiments, we test the impact of hand configuration properties and whether hand configuration properties can interfere with numerical processing even in high levels of expertise. Here, we postulate that handshapes representing closer numbers also share greater representational overlap, either visual or somatosensorial, and hence might interfere with processing the numerical information. To test this hypothesis, we use the handshape sequence for numbers in American Sign Language (ASL) to measure the influence of handshape similarity. The first advantage to using the ASL number signs is that all numbers are represented on one hand only (Figure 1). 
This means that there is a dissociation between the numerical meaning and the number of fingers raised for at least part of the sequence. In ASL, numbers 1 through 5 have a transparent representation of the numerical value: the number of fingers raised corresponds to the numerical value conveyed (i.e., iconic symbols). For ASL numbers 6 through 9, this correspondence does not hold as all are represented with only three fingers raised (i.e., abstract symbols). For example, 6 is represented with the thumb touching the little finger, 7 with the thumb touching the ring finger and so on (see Figure 1, bottom row). These abstract ASL number signs are not completely arbitrary as the thumb touches the opposing fingers in sequence, starting from the little finger to the index finger. Second, by using ASL number signs we are able to identify groups who vary in expertise in recognizing and using these handshapes. On the one
Figure 1

ASL Number Signs Used as Stimuli

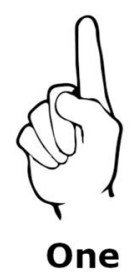

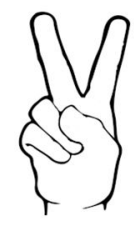

Two

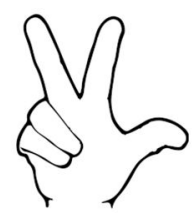

Three

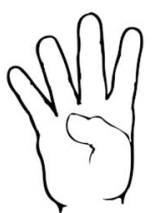

Four

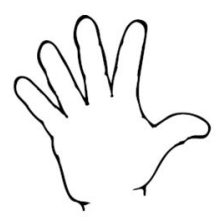

Five

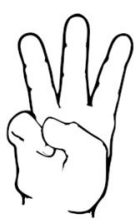

Six

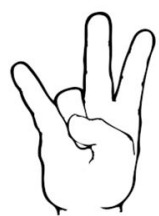

Seven

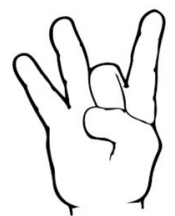

Eight

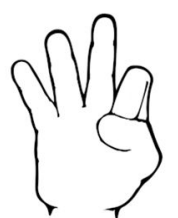

Nine
Note. For the signs on the top row, the number of fingers raised correlates with the numerical meaning represented. On the bottom row, ASL numbers signs 6 through 9 are all represented on one hand and there is no correspondence between number of fingers raised and numerical meaning represented.

Copyright: Graphics Factory. This figure is not permitted to be used outside this publication for commercial purposes.

end, hearing non-signers have used their fingers mostly for acquiring number concepts but are unfamiliar with at least part of the ASL number sequence. On the other end, we have Deaf native signers who are experts in processing handshapes and for whom ASL number signs are more than a support to acquire counting skills as these are linguistic symbols. Therefore, we can test if expertise in using and processing ASL numbers signs eliminates a possible influence of handshape similarity.

For the first time, in this study we will be able to test the impact of handshape by dissociating the physical properties of the stimuli from their numerical meaning, test whether handshape configurations interfere with the processing of the numerical information, and if expertise can eliminate the interference due to the similarity of the hand configurations. Last but not least, we test for the first time if the classical numerical distance effect can be observed in ASL number signs 1 through 9 as it has been observed for other number formats (e.g., Dehaene, Bossini, \& Giraux, 1993). So far, this has been only tested for the ASL numbers 1 through 5 where the number of fingers correspond to the number represented (Bull, Blatto-Vallee, \& Fabich, 2006). It is therefore impossible to know whether the effect was related to processing the number of fingers, just like processing sets of items, or if it was related to processing the handshapes as symbols. The latter can only be tested by including the ASL number signs above 5 as they are abstract symbols.

\section{Experiment 1}

In a group of hearing non-signers, we test the hypothesis that the handshape similarity can influence reaction time (RT) when asked to process the physical property regardless of whether numerical information is relevant. We specifically selected a group of participants unfamiliar with the ASL number signs as our aim was to test if processing handshapes carries its own pattern of responses. Therefore, participants were asked to make a same-different judgment on the physical properties of ASL handshapes. Participants were presented with pictures of pairs of ASL handshapes for number signs 1 through 9 and were asked to judge if these were the same or different. We first predicted the typical numerical distance effect for numbers up to five as observed with other formats for numbers (Dehaene et al., 1993). Indeed, processing quantities is automatic in educated adults and we predicted that comparing fingers raised could either be similar to comparing sets of items (e.g., dot comparisons; Gebuis, Cohen Kadosh, De Haan, \& Henik, 2009) 
or could rely on the numerical association of internalized finger counting strategies (Di Luca et al., 2010; Di Luca \& Pesenti, 2008). However, this result could also be related to the similarity observed between the handshapes. Therefore, because in the ASL number signs sequence there isn't a perfect match between number of fingers and the order in which fingers are raised (see Figure 1, ASL number signs 1 through 3) as well as a set of handshapes all with three fingers raised and two bent (i.e., ASL number signs 6 through 9), we also coded the number of common fingers for all the handshapes that plausibly represent numbers in our hearing non-signers group (i.e., we excluded handshapes for 7 and 8 as these are the only handshapes unlikely encountered in everyday life by non-signers). For example, ASL handshapes for numbers 1 and 2 have a numerical distance of one and one finger in common whereas ASL handshapes 2 and 3 have a numerical distance of one and two fingers in common just as ASL pairs 3 and 4 (see Figure 2, bottom row). By recoding ASL handshapes for numbers 6 and 9 as representing the numerical value of three in this group of non-signing participants, we were able to identify pairs that share up to four common fingers as well as a subset that shares up to three fingers with two numerical distances. Recoding handshapes that are not necessarily part of our participants' finger-counting sequence is likely to increase variability in performance. Therefore, if we do observe any effect, it will make our conclusions stronger on the influence of handshape similarity or on the automatic processing of numerical information if we observe a numerical distance effect.

We predicted that comparing handshapes would be increasingly hard with increasing shared properties which we operationalized by counting the number of common fingers. We also predicted an interaction where both variables, numerical value and the handshape similarity, would interfere with the same-different judgment. That is, trials where the handshapes share more common features as well as being numerically closer in the number of fingers raised, would be the ones showing the greatest interference. Additionally, to further test the influence of handshape similarity independently of numerical value, using the ASL handshapes for 3, and 6 through 9, all presenting three fingers raised (Figure 1, bottom row), we were able to test the hypothesis that the handshape similarity can influence reaction time and performance regardless of the actual numerical meaning conveyed by the number of fingers raised.

We first analyzed performance on the overall same-different judgment task to ensure participants complied with the task. Then analyzed only the pairs in the different condition for numbers 1 through 5 to test for the presence of what could be interpreted as the numerical distance effect even if no numerical judgement was required. To test for the influence of handshape, we analyzed the pairs based on the number of common fingers (four levels) and then the subset that varies on both numerical distance and number of fingers in common (Figure 2, bottom). Finally, we analyzed only the pairs for ASL handshapes 6 through 9 as they all have the same number of fingers raised but differ only by which finger is bent. To classify these pairs, we calculated the distance between the fingers bent given that all share the same number of common fingers (i.e., two). Therefore, ASL handshapes for 6 (little finger bent) and 7 (ring finger bent), should be harder to discriminate than ASL handshapes for 6 (little finger bent) and 8 (middle finger bent). This classification also allows for opposite predictions based on whether performance is driven by handshape or numerical processing. Based on the handshape similarity, ASL handshapes for 6 and 9 should be the easiest as the fingers bent are the most distant. However, based on the numerical representation, both handshapes should convey the numerical meaning of three as they are plausible handshapes encountered by the non-signing participants (Bender \& Beller, 2012, for examples of culturally used counting sequences). Therefore, comparison should be harder as the numerical information should slow down the response process as well as prime a 'same', rather than 'different', response selection. On the contrary, ASL handshapes for 7 and 8 are atypical configurations, most likely never encountered by hearing non-signers. If performance is driven by automatic access of numerical information, these have weaker associations (i.e., Di Luca \& Pesenti, 2008; Di Luca et al., 2010) and should result in higher performance. If performance is driven by handshape similarity, these ASL handshapes for 7 and 8 should appear harder to discriminate as the bent fingers are closer to each other. To ensure that results for this analysis were not due to little familiarity with the ASL handshape for 9 to represent the numerical quantity of three, we also ran the comparison with the ASL handshapes 3 and 6 . The ASL handshape for 3 (thumb, index and middle fingers) is more commonly used and the pair still shares two common fingers, therefore keeping the predictions unchanged. 


\section{Method}

\section{Participants}

Thirty hearing participants who had never been exposed to ASL or had only very minimal exposure to ASL were recruited from the Washington DC metro area. The majority of participants were right-handed (28 out of 30); one participant identified as being ambidextrous and one as left-handed. No participants reported any history of specific learning disability nor suffered any major neurological disorder. Age range was between 19 and 37, with a mean of 27 years and 6 months. Ten participants identified as male. Participants were compensated for their time and the study was approved by the Institutional Review Board.

\section{Procedure and Stimuli}

This experiment was part of a larger study including several numerical tasks. As the other tasks relate to separate questions and hypotheses, those results will not be discussed here. Hearing participants were greeted and instructed to the tasks by an English-speaking research assistant. All tasks were presented on a laptop running Windows 10 using E-Prime 3 software. The background questionnaire on language and demographic information was administered through RedCap.

Stimuli were black line drawings of ASL handshapes for numbers 1 through 9 with hand orientation showing the palm (Figure 1). Participants were asked to determine as quickly and accurately as possible if two handshapes were the same or different pressing the letters "f" and " $\mathrm{j}$ " on a keyboard. Response keys were counterbalanced across participants. All ASL handshapes were repeated eight times for 'same-trials' (e.g., the ASL handshape for number 1 paired with the same ASL handshape and so on for a total of 72 stimuli: 9 ASL handshapes $x 8$ repetitions) and all other combinations of handshapes were presented three times constituting the 'different-trials' (9 ASL handshapes $\mathrm{x} 8$ possible combinations x 3 repetitions for a total of 216 stimuli). The entire experiment included 288 stimuli (216 'different-trials' plus 72 'same-trials') divided into 3 blocks of 96 trials each.

The experiment started with an instruction screen explaining the task and response key mapping for 'same' and 'different' responses. A block started with a $200 \mathrm{~ms}$ fixation and then the pair of handshapes remained on the screen until a response was given. During breaks, participants could rest for as long as they needed.

\section{Data Analyses}

Of the 30 participants, two were administered the wrong task and six performed below $75 \%$ accuracy either because they forgot the response key mapping or because they disengaged from the task. Therefore, only 22 hearing non-signers were retained for the analyses. All trials beyond 2.5 SD have been removed from RT averages and accuracy percentages. Response times are averaged over correct trials only. In all subsequent analyses, response times (RT) were analyzed using repeated measures ANOVAS and the Huynh-Feldt correction was applied when the assumption of sphericity was not met.

\section{Results}

\section{Same-Different Analyses}

Same and different judgments were made equally fast, $F(1,21)=2.52, p>.05$. Accuracy was significantly lower for 'same-trials', $F(1,21)=31.73, p<.001, \eta_{\mathrm{p}}^{2}=.60$, but overall, performance was high ('same-trials': $92.8 \%$ and $S E M=0.4 \%$; 'different-trials': $97.9 \%$ and $S E M=1.1 \%$ ).

\section{Numerical Distance Effect}

For ASL handshapes between 1 and 5, we found a numerical distance effect for RTs, $F(2.3,48.35)=5.11, p=.007$, $\eta_{\mathrm{p}}^{2}=.20$, but not for accuracy, $F(3,63)=.99, p>.05$. RTs show the typical increase in judgment time for pairs with closer numerical values. For distances 1 through 4, RT averages were: $\mu(S E M)=827(33) \mathrm{ms}, 773(29) \mathrm{ms}, 752(28) \mathrm{ms}$, and $742(27) \mathrm{ms}$. Both linear and quadratic trends were significant $\left(p s<.05, \eta_{\mathrm{p}}^{2}=.27\right.$ and .21 for linear and quadratic trends, 
respectively). This confirms that our similarity judgment task elicits a result that can be interpreted in terms of the typical numerical distance effect even when numerical judgment is not required.

\section{Handshape Similarity Effect}

All pairs, except those including ASL handshapes for 7 and 8, were coded based on the number of common fingers in each pair. The analysis on the number of common fingers (4 levels) returned a significant effect for accuracies and RTs, $F(1.9,39.97)=3.77, p=.034, \eta_{\mathrm{p}}^{2}=.15$ and $F(1,41.81)=9.01, p=.005, \eta_{\mathrm{p}}^{2}=.30$, respectively. Accuracy decreased and RTs increased as the number of fingers in common increased (Figure 2, top left). For both, the linear trend was significant ( $p s$ $<.05)$.

Further, in a subsequent analysis, we included number of fingers in common (3 levels) and numerical distance (2 levels). For both accuracy and RT, we found both main effects and the interaction to be significant (Figure 2, top right). With increased number of common fingers, performance decreased in accuracy and speed, $F(2,42)=13.56, p<.001$, $\eta_{\mathrm{p}}^{2}=.39$, and $F(2,42)=29.27, p<.001, \eta_{\mathrm{p}}^{2}=.58$ for accuracy and RT, respectively. Performance also significantly decreased for the numerical distance of 1 compared to $2, F(1,21)=17.40, p<.001, \eta_{\mathrm{p}}^{2}=.45$, and $F(1,21)=36.95, p<.001, \eta_{\mathrm{p}}^{2}=.64$ for accuracy and RT, respectively. The interaction showed that for pairs with a numerical distance of 1 , performance was impacted more with increasing number of common fingers, $F(1.47,30.94)=15.54, p<.001, \eta_{\mathrm{p}}^{2}=.43$, and $F(2,42)=15.06, p$ $<.001, \eta_{\mathrm{p}}^{2}=.42$ for accuracy and RT, respectively.

Figure 2

Results for the Hearing Non-Signing Group

Shared Fingers Effect

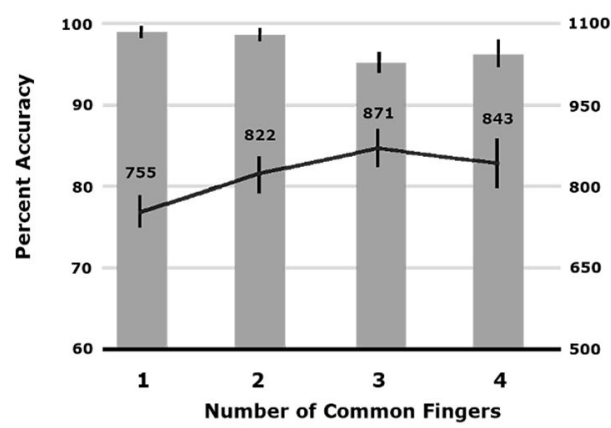

Numerical Distance by Number of Common Fingers

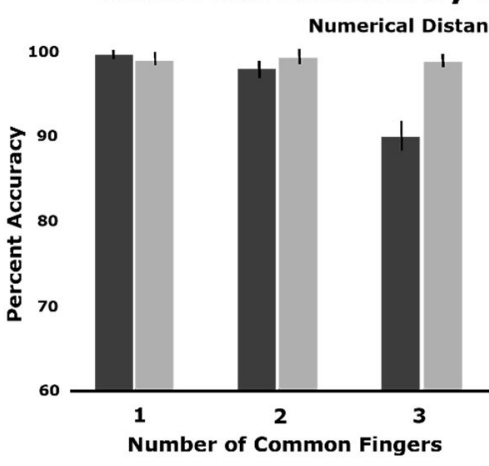

1 - 2 -

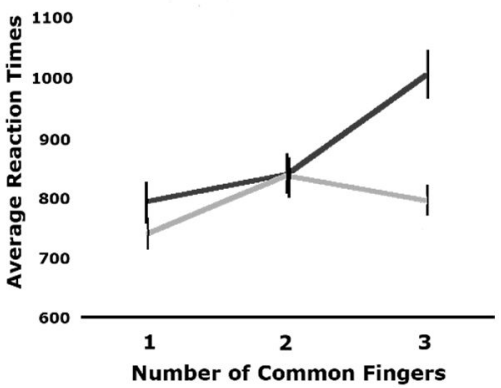

\begin{tabular}{|c|c|c|c|}
\hline & \multicolumn{3}{|c|}{ Number of Common Fingers } \\
\hline & 1 & 2 & 3 \\
\hline 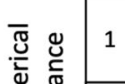 & $\begin{array}{l}(1-22-9) \\
\text { A } M\end{array}$ & $\begin{array}{l}(2-32-63-4) \\
M M \\
M M\end{array}$ & (4-6 4-9) \\
\hline 2 & $\begin{array}{l}(1-31-6) \\
\text { की }\end{array}$ & $M_{M}^{(2-4)}$ & 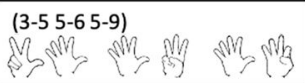 \\
\hline
\end{tabular}

Note. Graphs present results for the hearing non-signing group based on the number of fingers shared in the ASL handshapes. Top left panel shows percent accuracy and average RTs (ms) by number of fingers in common in each pair for ASL handshapes 1 through 5. The top right panel shows the interaction for percent accuracy and average RTs for a subset of stimuli that varied orthogonally for number of common fingers (1,2 or 3$)$ and numerical distance (1 and 2). Bars represent SEM. The bottom panel shows all the pairs coded for numerical distance and number of fingers in common. For all the handshape pairs, the corresponding ASL numbers are shown in parentheses.

Copyright: Graphics Factory. This figure is not permitted to be used outside this publication for commercial purposes.

It is important to remember that for ASL handshapes 6 through 9 used in the analysis, hearing non-signers could only extract the information provided by the number of fingers raised, in this case always three. Any difference observed in 
the next analysis cannot be attributed to numerical meaning or number of fingers in common as it is always the same for all pairs. Differences can only be attributed to the difficulty in processing handshapes based on the distance between fingers bent.

We found a finger distance effect for RT and accuracies, where pairs with adjacent fingers bent (e.g., pair of ASL handshapes 8-9, see Figure 1) were the hardest and pairs where bent fingers had a distances of 2 fingers (e.g., 7-9) and 3 fingers (e.g., 6-9) were comparable, $F(1.52,31.83)=14.89, p<.001, \eta_{\mathrm{p}}^{2}=.42$ and $F(2,42)=6.24, p=.004, \eta_{\mathrm{p}}^{2}=.23$, for accuracy and RTs, respectively. For pairs of handshapes in which adjacent fingers were bent, accuracy was $93.4 \%$ (SEM = $1.4 \%)$ and RT was $984 \mathrm{~ms}(S E M=40 \mathrm{~ms})$. For pairs of handshapes in which bent fingers had a distance of 2 or 3 fingers, accuracies were $99.6 \%(S E M=0.4 \%)$ and $99.2 \%($ SEM $=0.8 \%)$, respectively; RTs were $869 \mathrm{~ms}(S E M=27 \mathrm{~ms})$ and $874 \mathrm{~ms}$ $(S E M=38 \mathrm{~ms})$, respectively. A linear trend was significant only for RTs $\left(p=.011, \eta_{\mathrm{p}}^{2}=.27\right)$.

Finally, we tested the pairs of handshapes that provide opposite predictions based on whether numerical or handshape similarity influence performance. In this analysis, we compared performance for the pair of handshapes for 6 and 9, acceptable handshapes for three, with the ASL handshapes for 7 and 8, that are atypical handshapes for three. Results indicate that comparing ASL handshapes for 7 and 8 was harder, $F(1,21)=10.44, p=.004, \eta_{\mathrm{p}}^{2}=.33$, and slower, $F(1,21)=12.7, p=.002, \eta_{\mathrm{p}}^{2}=.38$, than comparing the ASL handshapes for 6 and 9. Accuracies and RTs were: $99.2 \%$ $(S E M=0.8 \%)$ and $874 \mathrm{~ms}(S E M=38 \mathrm{~ms})$ for ASL handshapes 6 and 9 ; and $88.3 \%(S E M=0.3 \%)$ and $1071 \mathrm{~ms}(S E M=62 \mathrm{~ms})$ for ASL handshapes 7 and 8. This suggests that numerical information did not interfere but that ASL handshapes 7 and 8 were harder due to the proximity of the fingers bent. Because one could argue that the ASL handshape for 9 is not as frequent as other representations of the numerical quantity 3, among the different ASL handshapes, we identified the ASL handshape for 3 which is more commonly used than the handshape 9. Therefore, we selected the pair of ASL handshapes 3 and 6 which also shares 2 common fingers just like the pair 7 and 8, thus making it a suitable pair to test our hypothesis while keeping the predictions unchanged. Results show the same outcome where handshapes for 7 and 8 were harder, $F(1,21)=11.67, p=.003, \eta_{p}^{2}=.36,99 \%$ vs $88 \%$, and slower, $F(1,21)=35.21, p<.001, \eta_{p}^{2}=.63,834 \mathrm{~ms}$ vs $1070 \mathrm{~ms}$.

\section{Discussion of Experiment 1}

In experiment 1, we asked non-signers to make a same-different judgment on pairs of handshapes used in ASL to represent numbers. We found a numerical distance effect although participants were not required to process numerical information. This result is in line with studies showing automatic processing of numerical information with different numerical formats (Gebuis et al., 2009; Henik \& Tzelgov, 1982). We also found that performance is influenced by the similarity between handshapes both due to the number of fingers shared or due to the distance between the fingers bent. These results suggest that processing numerical information presented on the hands is not independent of other perceptual information. Other number formats such as Arabic digits and number words bear an abstract relationship between the numerical meaning and the perceptual qualities of their format. The Arabic digit or the spoken/written number word for two does not transparently convey the meaning of 'twoness' and it does not build on the prior symbols. Therefore, a confound between the format and the numerical meaning appears only for the finger counting sequence.

Interestingly, numerical distance appears to modulate the same-different judgment most when the numerical values are closer. Indeed, accuracy was lowest and RTs longest when the pairs shared three common fingers and had a numerical distance of one. Because the two dimensions appear to be processed with some level of dependence, the interaction could occur at different levels in the processing stream: representational or decisional level (see Santens \& Verguts, 2011; Verguts \& Fias, 2008; for a similar reasoning regarding the size congruity interaction). For the interaction to occur at the representational level, this would mean that both the numerical and handshape information are mapped onto a shared representation. For an interaction to occur at the decision level, this would mean that both dimensions are processed independently up to the decisional level, regardless of whether the numerical information is relevant for the task. Up to now, different models for numerical representation have been proposed with some arguing for a shared representation for different quantitative dimensions (e.g., the ATOM theory by Walsh, 2003). However, none has proposed that somatosensory representation be one of these dimensions. A few neuroimaging studies have found 
overlapping activations for numerical and hand processing (Andres, Seron, \& Olivier, 2007; Sato, Cattaneo, Rizzolatti, \& Gallese, 2007) whereas others have found activations for finger-based strategies to occur in somatosensory and pre-motor areas while numerical processing is typically known to occur in the intraparietal sulci (Andres, Michaux, \& Pesenti, 2012; Berteletti \& Booth, 2015; Krinzinger et al., 2011). An interesting future question would be to understand if our stimuli do indeed recruit somatosensory representations and if the interaction between the amount of overlapping hand representation and overlapping numerical representation occurs at a shared or independent representational level.

Another interesting observation is a possible "full-hand" effect. Considering the analysis on shared number of fingers, performance appears to rebound for a distance of 4. Similarly, for the interaction between shared fingers and numerical distance, RTs appear to decrease for three shared fingers and a numerical distance of two. In both these situations, one of the handshapes being compared is always the ASL handshape for 5 (i.e., the full hand; Figure 2, bottom row). This handshape could be more easily recognizable and facilitate the similarity judgment.

The following question is whether extensive practice and the explicit request to process the numerical information can counter the handshape similarity effect as the attention is now shifted from the handshape properties to the numerical information conveyed. Can the processing of handshapes become detached from their superficial perceptual features when these are symbols used as part of a language system and not merely as a tool to acquire numerical understandings?

\section{Experiment 2}

In a group of Deaf ASL native signers, we first tested and predicted a numerical distance effect for all ASL numbers signs 1 through 9 since these signs are numerical symbols equivalent to any other number format. Bull and colleagues (Bull et al., 2006) tested the numerical distance effect in a numerical Stroop paradigm with ASL handshapes for numbers 1 through 5 in both hearing and Deaf participants. They found that for this range, where the ASL number signs have a direct correspondence between the number of fingers raised and the numerical values represented, both groups showed a numerical distance effect. However, this result could be considered similar to results found when comparing sets of items. The interesting part of the ASL number sequence lies within its abstract symbols for numbers beyond 5. To the best of our knowledge, no study has empirically tested whether Deaf ASL native signers show a numerical distance effect for ASL numbers 1 through 9. Additionally, we were also able to orthogonally classify a subset of number pairs based on their numerical value and based on the number of overlapping fingers, allowing us to test the influence of both number and handshape similarity on performance. In this group, compared to the hearing non-signer group, we were able to identify a larger set of pairs with distances up to 5 as we included all ASL number signs between 1 and 9. Therefore, in experiment 2 we wanted to further test whether handshape similarity can interfere with the numerical processing even in expert signers for whom ASL number signs are strongly trained symbols and part of a language system.

Deaf native signers were asked to judge which of two ASL number signs was numerically larger to ensure that they were retrieving numerical information and stack the deck against a more cognitively superficial perceptual processing strategy. Importantly, the aim was to evaluate how handshape interferes with numerical processing and not the reverse. We first analyzed the numerical distance effect to test the prediction that ASL number signs, regardless of whether they are transparent ( 1 through 5 ) or not (6 and above), are comparable to other number formats by showing the classical numerical distance effect (Dehaene et al., 1993). Then, to understand if handshape configuration also influences performance in the ASL number judgment task, we classified ASL number pairs based on the number of common fingers raised as well as their actual numerical meaning. We were able to identify 29 pairs with 1 , 2 or 3 fingers in common that varied orthogonally in their numerical distance, that is 1 through 5 (see Figure 3). There was no correlation between numerical distance and number of common fingers $(r=-.077)$. 


\section{Method}

\section{Participants}

Thirty-six Deaf native signers were recruited for this study. All declared having been exposed to ASL prior to age two and have received the majority of their instruction in ASL. All reported using ASL on a daily basis and were recruited from the DC metro area where a sizeable Deaf-ASL signing community thrives. Four participants declared being lefthanded and two ambidextrous, whereas all others reported being right-handed. Five participants were excluded due to their report of prior Attention Deficit and Hyperactivity Disorder or other learning disability. Age range for the 31 participants retained for the analyses was between 18 and 32, with a mean of 23 years and 6 months. Eleven participants identified as male and 20 as female. Participants were compensated for their time and the study was approved by the Institutional Review Board.

\section{Procedure and Stimuli}

This experiment was part of a larger battery of numerical tasks administered to both the hearing non-signer group and the Deaf native signers. For optimal communication with our Deaf signing group, the research assistant leading this study was also a Deaf native ASL signer. All tasks were presented on a laptop running Windows 10 using E-Prime 3 software. Stimuli were black line drawings of ASL number signs 1 through 9 (Figure 1). Participants were asked to determine as quickly and accurately as possible which of two ASL number signs was numerically larger by pressing the ipsilateral key "f" or " $\mathrm{j}$ " on a keyboard. All combinations of numbers (i.e., 36 pairs) were presented eight times with the side of the largest number counterbalanced for half the trials. In total, participants responded to 288 stimuli divided into 3 blocks of 96 trials each.

The experiment started with an instruction screen explaining the task and the keys for responding. A block started with a fixation for $200 \mathrm{~ms}$ and then the pair of ASL number signs remained on the screen until a response was given. Participants could rest as long as they wanted between blocks.

\section{Data Analysis}

All 31 participants were retained for the analyses as overall accuracy was over $88 \%$. All trials beyond $2.5 S D$ were removed from RT averages and accuracy percentages. Response times were averaged over correct trials only. Data was analyzed with repeated measures ANOVAs and the Huynh-Feldt correction was applied when the assumption of sphericity was not met.

\section{Figure 3}

Pairs of ASL Number Signs Included in the Distance x Common Finger Analysis

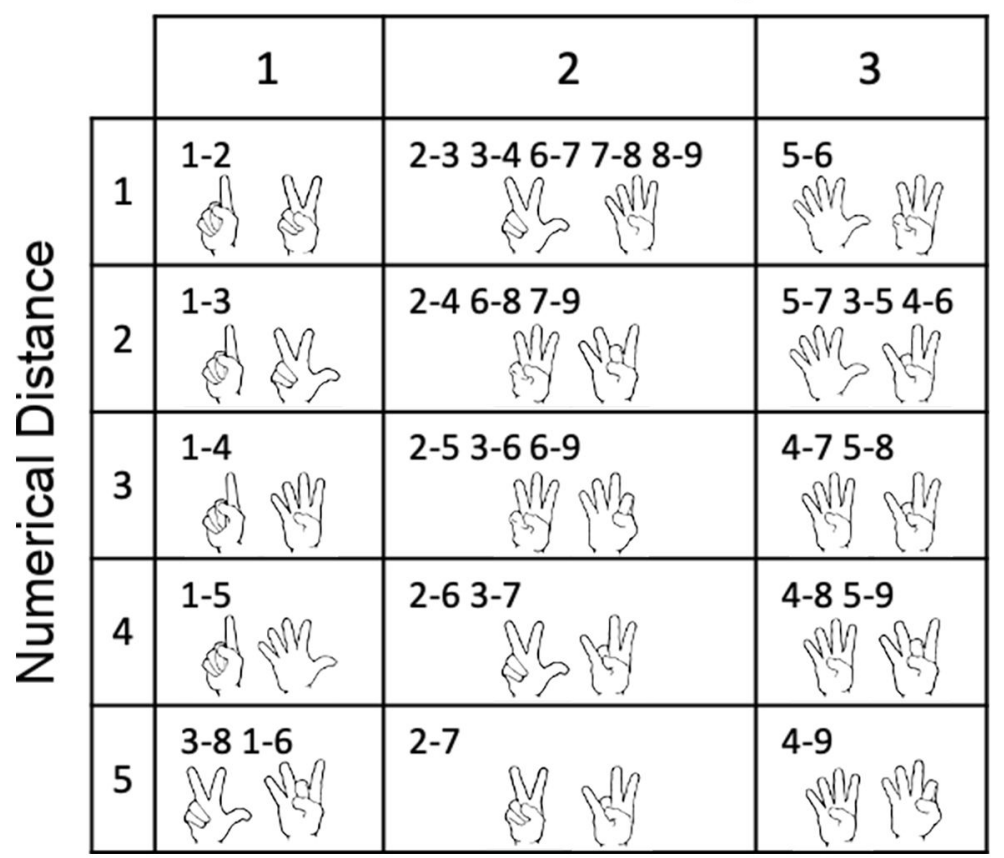

Note. Twenty-nine pairs were reclassified based on their numerical distance (1 through 5$)$ and the number of common fingers raised (1 through 3). All possible pairs for each combination of numerical distance and common fingers are presented. The numerical pair printed in bold type is also represented the corresponding handshapes as an example for each cell.

Copyright: Graphics Factory. This figure is not permitted to be used outside this publication for commercial purposes. 


\section{Results}

\section{Numerical Distance Effect for ASL Number Signs}

Performance for determining the larger of two ASL numbers signs showed the expected numerical distance effect for both accuracy and RTs, $F(3.52,105.55)=15.65, p<.001, \eta_{\mathrm{p}}^{2}=.34$ and $F(5.49,164.65)=41.73, p<.001, \eta_{\mathrm{p}}^{2}=.58$, see Figure 4. For accuracy, both linear and quadratic trends were significant $(p s<.001)$, indicative of a ceiling effect for larger distances, whereas only the linear trend was significant for the RTs, indicative of steadily decreasing RTs with increasing numerical distance $(p<.001)$.

We also analyzed the data for ASL numbers signs 6 through 9 as Figure 4 these ASL numbers do not represent the numerical quantity through a corresponding number of fingers raised but are non-transparent symbols. Again, performance showed a numerical distance effect for both accuracy, $F(2,60)=10.93, p<.001, \eta_{\mathrm{p}}^{2}$ $=.27$, and RTs, $F(1.62,48.53)=11.10, p$ $<.001, \eta_{\mathrm{p}}^{2}=.27$. Both linear and quadratic trends were significant for accuracy (linear: $p<.001$, and quadratic: $p$ $<.01)$ and only the linear trend was significant for RTs $(p<.001)$.

\section{Numerical Distance vs. Handshape Similarity}

In this analysis we entered numeriNumerical Distance Effect in the Deaf ASL Native Signing Group

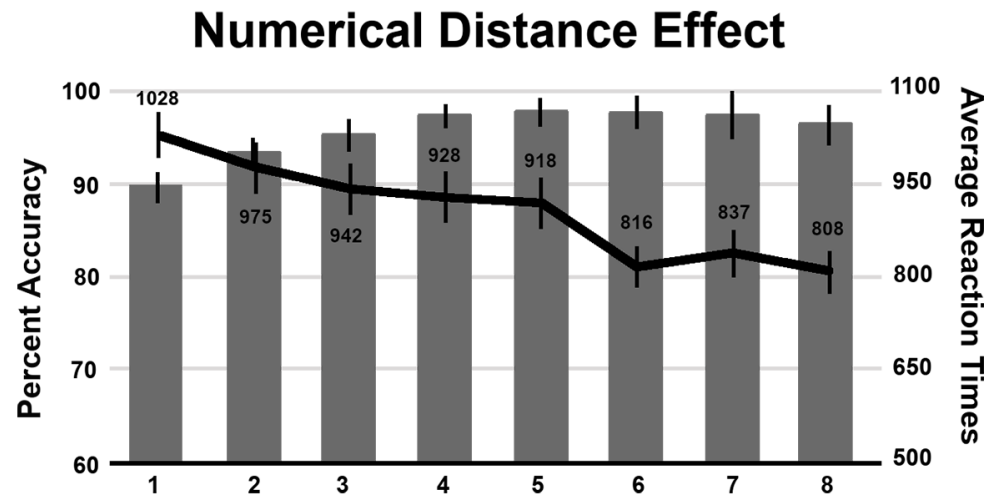

Note. Percent accuracy and average RTs (ms) for comparing ASL number signs based on numerical distance in a group of Deaf native signers. Bars represent SEM. Accuracies increase and RTs decrease with increase numerical distance between the two ASL numbers, effect consistent with what is observed with other number formats.

Copyright: Graphics Factory. This figure is not permitted to be used outside this publication for commercial purposes.

cal distance (5 levels) and number of

common fingers (3 levels) as variables. For accuracies and RTs, both main effects and the interaction were significant (Figure 5). Accuracies were lower and RTs were longer for pairs with more common fingers, $F(2,60)=18.39, p<.001, \eta_{p}^{2}$ $=.38$ and $F(1.4,46.06)=46.78, p<.001, \eta_{\mathrm{p}}^{2}=.61$, respectively. With increasing numerical distances performance was more accurate and faster, $F(4,120)=23.86, p<.001, \eta_{\mathrm{p}}^{2}=.44$ and $F(4,120)=8.02, p<.001, \eta_{\mathrm{p}}^{2}=.21$, respectively.

Crucially, the interaction showed that the impact of common fingers was greater for numerically closer pairs than numerically more distant pairs, $F(3.75,112.6)=5.33, p<.001, \eta_{\mathrm{p}}^{2}=.15$ and $F(8,240)=5.08, p<.001, \eta_{\mathrm{p}}^{2}=.15$, for accuracy and RT, respectively. Accuracies were lower and RTs slower for numerically closer pairs sharing more fingers. 


\section{Discussion of Experiment 2}

The first relevant result from Experiment 2 , is that ASL numbers signs induce the classical numerical distance effect, as widely documented in all other number formats. Even when only considering the subset of ASL signs for numbers 6 through 9, which are abstract symbols. This is the first time it has been tested with handshape configurations representing all numbers on one single hand. The second result is that handshape similarity has a significant influence on the processing of the ASL number signs where signs that share more common hand features, that is share more common fingers raised, are harder to process than those that share less features. Therefore, even in a group of individuals with extensive training, who communicates primarily through the use of hands, the superficial features of the handshape interfere with the processing of the numerical information.

Again, we see that the processing for the two dimensions occur with a certain level of dependence. Because these handshapes constitute linguistic symbols for ASL native signers, an interesting question is whether the interference occurs at a different level compared to the one observed in hearing participants.

Figure 5

Numerical Distance by Common Fingers in the Deaf ASL Native Signing Group
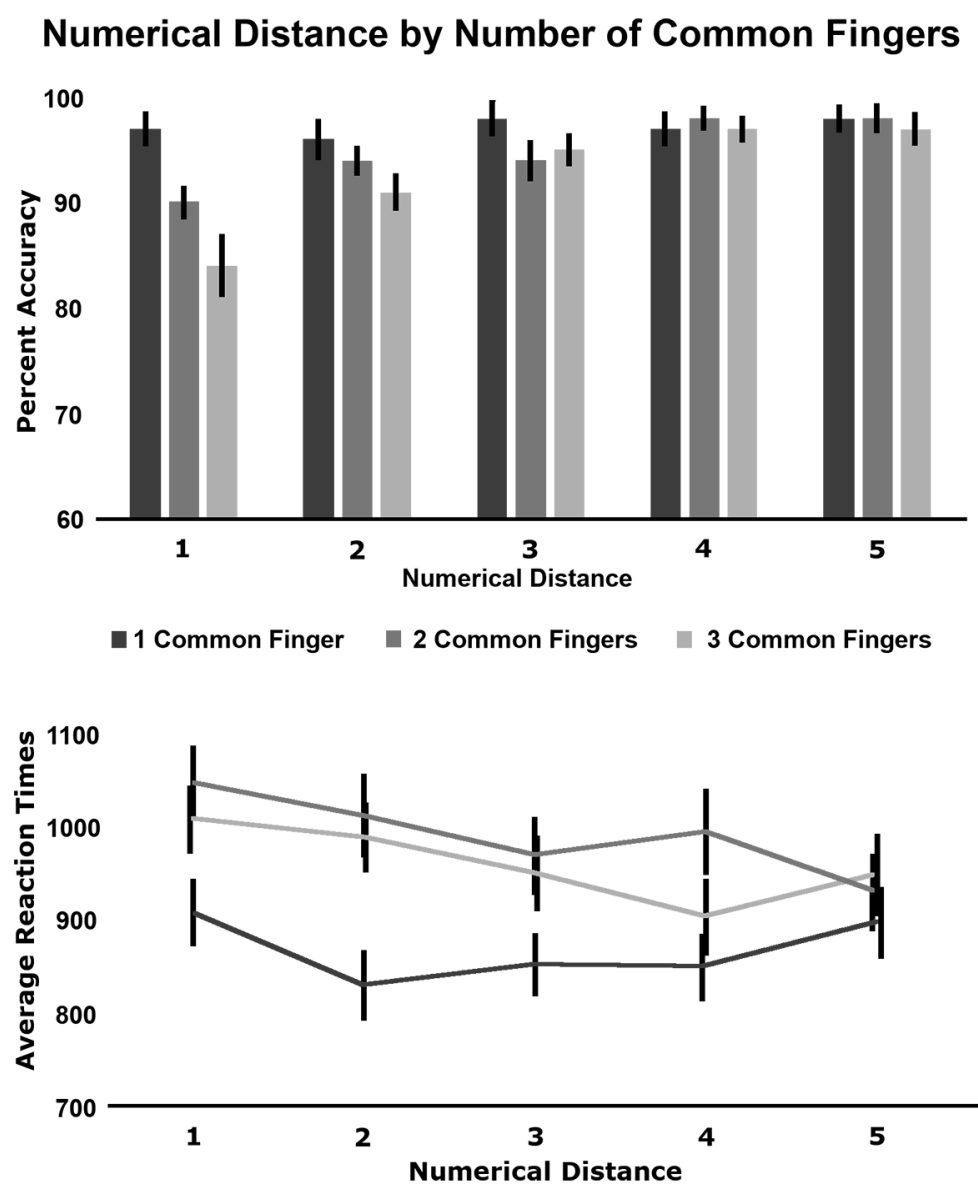

Note. Percent accuracy (top) and average RTs (bottom) in milliseconds for the interaction between numerical distance and number of fingers in common when Deaf native signers compare two ASL number signs. For smaller numerical distances, sharing more fingers decreases accuracy and increases RTs. For larger distances, the impact of the number of common fingers diminishes. Interestingly, RTs for pairs sharing three common fingers appear faster than those sharing 2 common fingers. This could be due to the greater occurrence of the ASL number sign for 5, which corresponds to the "full hand" (i.e., all five fingers raised). This handshape could be recognized faster. However, only RTs appear to be influenced.

Copyright: Graphics Factory. This figure is not permitted to be used outside this publication for commercial purposes.

\section{General Discussion}

In these two experiments, we tested whether the handshapes for representing numbers can carry its own processing effects and if it can interfere with the numerical processing when part of a symbolic system. Answering this question is relevant given that often finger-based strategies are used to teach children how to count and given the observation that processing numerical information appears to rely on or recall these internalized hand configurations and finger-based strategies (Andres \& Pesenti, 2015; Berteletti \& Booth, 2016 for comprehensive reviews).

In these two studies, we show several effects. In our first experiment with hearing non-signing participants, we replicate a numerical distance effect for pairs of iconic handshapes (where the number of fingers raised represents the numerical value) as observed in a Stroop-like paradigm by Bull and colleagues (2006). This is consistent with the numerical distance effect observed with several other number formats (Dehaene et al., 1993). However, we also show 
for the first time a handshape similarity effect where greater similarity between the handshapes negatively impacted performance. The role of handshape similarity was confirmed with two analyses involving a subset of handshapes (ASL handshapes for numbers 3, and 6 through 9). Difficulty was not related to implicit numerical processing but to the ability to distinguish handshapes.

Further, implicit numerical information also interfered with handshape processing. When the pairs of handshapes shared more common features, and thus were harder to discriminate, the influence of numerical distance was greater. This suggests that the two dimensions are not completely independent. An interesting question relates to the locus of the interference. Some studies have identified common brain areas responsible for processing both dimensions (Andres et al., 2007; Sato et al., 2007), others have found separate brain areas (Berteletti \& Booth, 2015; Krinzinger et al., 2011). Additionally, because the handshapes used here are functionally different for our two groups, a counting tool versus a linguistic symbol, the question is whether the interference in these two groups might occur at different levels in the processing stage and through different cognitive mechanisms.

In our second experiment, we also find another set of novel findings with our group of Deaf ASL native signers. First, we show for the first time that handshapes for numbers presented on one hand, ASL number signs 1 through 9, elicit the classical numerical distance effect even when the handshapes used have no transparent correspondence between the number of fingers raised and the numerical meaning conveyed. The previous study by Bull and colleagues (2006) only tested handshapes 1 through 5 where numerical information is transparently conveyed on the hands. Although this appears to be a self-evident result given that sign languages, such as ASL, have been already demonstrated to be equivalent to any other spoken language, it still remains relevant as it has not yet been demonstrated. Consequently, this confirms that all number formats, expressed in any modality, do indeed yield the same numerical distance effect. Because evidence shows that delayed access to a full language during critical periods of language acquisition impacts later reading skills (Padden \& Ramsey, 2000), it would be relevant to investigate how delayed access to a full visual language might impact ASL number sign processing and the strength among the different number formats. With this result, we also establish a baseline of proficient ASL number processing in our group of native (i.e., early exposed) ASL signers to then investigate specific math learning disabilities in Deaf signers. Future studies should investigate whether performance on an ASL number sign comparison task relates to arithmetic performance in Deaf signing children as it has been observed in hearing children with symbolic numbers (Holloway \& Ansari, 2009), and whether this relationship is modulated by age of sign language acquisition.

The second relevant result observed in the experiment with Deaf native signers is that expertise in using ASL number signs as part of one's language system does not prevent the interference caused by handshape similarity. Indeed, there was an effect of handshape similarity and it was stronger with decreasing numerical distance. It is relevant to highlight that our stimuli used here are far from what a Deaf signer experiences during everyday linguistic interactions. Hence, it remains to be determined if this influence is related to the format in which the stimuli were presented in this experiment; Deaf signers usually process handshapes while in movement and in a linguistic context, not fixed hand drawings.

The results from the two experiments both agree in showing how handshape similarity has its own effect and that it can interfere with numerical information processing as well as being subject to numerical interference. This brings new insights to the possible relationship observed between finger gnosis and arithmetic proficiency in children (Fayol et al., 1998; Newman, 2016; Noël, 2005; Reeve \& Humberstone, 2011). The first interpretation is that poor finger gnosis prevents children from easily distinguishing hand configurations that are more similar. Because most counting sequences show a strong correlation between the number of fingers raised and the numerical meaning, closer numerical configurations also show greater handshape similarity making the distinction even harder. The second possible non-mutually exclusive interpretation is that poor finger gnosis in children is a consequence of failed training on one's fingers. Chinello and colleagues (Chinello, Cattani, Bonfiglioli, Dehaene, \& Piazza, 2013) suggest that there could be a possible reciprocal training between counting, using hand configuration sequences, and finger gnosis. Therefore, if a child fails in accurately recognizing handshapes, they will benefit less from the visual support provided by adults and peers showing how to count and calculate using their fingers. Till this day, the relationship between finger gnosis and arithmetical proficiency is still poorly understood (Soylu, Raymond, Gutierrez, \& Newman, 2018) and indeed, not all children need to rely on finger counting to succeed on numerical tasks (Lafay et al., 2013). 
These results also provide new directions for reinterpreting the findings showing a relationship between internal representations of hand configurations and numerical processing. Maybe some of the differences observed in behavioral studies using canonical patterns and non-canonical patterns could also be explained by the observation that canonical patterns are usually sequences of consecutive fingers being raised for each successive numerical value whereas non-canonical patters are often hand configurations that deviate from this succession (e.g., Di Luca \& Pesenti, 2008; Di Luca et al., 2010; Nicoladis et al., 2018; for studies using canonical and non-canonical handshapes). Future studies should consider controlling for this variable especially as both numerical and handshape similarity may produce very similar distance effects.

Studies with hearing participants have subdivided canonical hand configurations into montring and counting sub categories. Montring refers to the handshapes used to show a cardinal amount whereas the counting handshapes refer to the sequence used when counting items (Di Luca \& Pesenti, 2008; Di Luca et al., 2010). Both montring and counting have shown to be qualitatively different from noncanonical configurations (Di Luca \& Pesenti, 2008; Di Luca et al., 2010) but behavioral and neural differences have also been found between montring and counting: montring handshapes were processed faster but also recruited greater attentional resources (Di Luca et al., 2010; Soylu, Rivera, Anchan, \& Shannon, 2019). Although the current study does not allow to test differences between the subcategories, it does raise the question on whether results would be different depending on hand orientation for the ASL signing participants. In ASL, hand orientation and hand movement convey different meanings. The sequence of handshapes does not change but the palm orientation towards the signer indicates cardinality whereas palm orientation towards the interlocutor is used for labels. Movement can also express rank or ordinality. For example, if an ASL signer was to express how many apples are in a basket, the palm would be oriented inwards; if the signer were to communicate a phone number, the palm would be oriented outwards. For describing that a runner was $3^{\text {rd }}$, palm orientation would go from outward to inward. Our current stimuli, showing inward palm orientation, are consistent with an egocentric view of a cardinality representation. It would be interesting to test whether stimuli showing the back of the hand, static or in movement, also produce the same effects ${ }^{1}$.

If we know that these handshapes are the canonical representation for our Deaf signing group, we unfortunately don't have such information for our hearing participants. Our results are only based on the assumption that this group of English-speaking participants were exposed to the culturally-canonical counting sequence. The lack of this information does not however necessarily weaken the findings. Our results show a numerical distance effect regardless of whether the handshapes were part of the canonical pattern and we see an interference effect of numerical information on the handshape similarity suggesting that numerical information was automatically processed. We can conclude that the handshapes were either familiar enough to our group of participants or, if they were not part of their canonical configurations, these still provided automatic access to numerical information for an interference to occur.

The other interesting question that is raised here is whether these stimuli presented as handshapes automatically activated an internal representation of the body and if the interference observed occurs at a visual-perceptual level or at a somatosensory level. If processing numbers and calculation have shown activations in somatosensory areas for the fingers in the brain (Berteletti \& Booth, 2015), it seems plausible that processing handshapes representing numbers would also activate brain areas for somatosensory and motor processing. Indeed, studies have shown the existence of specific visual areas dedicated to the processing of body parts, such as the bilateral extrastriate body area (EBA) as well as the post-central gyrus (i.e., the somatosensory areas), and these activate specifically when presented with images of the body (Downing \& Kanwisher, 2001; Saxe, Jamal, \& Powell, 2006; Taylor-Clarke, Kennett, \& Haggard, 2002). A separate line of research on sign language processing has shown that signers rely primarily on somatosensory, rather than visual, feedback when monitoring language output (Emmorey, Bosworth, \& Kraljic, 2009). Therefore, it is possible that processing pictures of hand configurations for numbers also activates both representations. Future studies should investigate whether seeing handshapes for numbers does indeed activate the somatosensory representation and if the

1) In a personal communication with a teacher from a local Elementary School for the Deaf, it appears that children are encouraged to use the same ASL number signs for both counting, calculating and montring. The teacher reported that this was done to reduce the amount of possible confusion, especially for ASL numbers above 5 . 
observed effect stems from shared or distinct representations. Such result would indeed further clarify the observed relationship between finger gnosis and numerical processing abilities.

In conclusion, in this study we show an impact of handshape similarity for processing handshape configurations used for counting. Even in a group of highly experienced Deaf native signers, handshape similarity interfered with the numerical processing of the ASL number signs. This suggests that handshapes are qualitatively different from other number formats and might carry their own specific difficulties when used as support for learning and teaching numerical concepts as well as linguistic symbols in sign languages.

Funding: This project was supported by Gallaudet University and the NSF/Gallaudet University's Science of Learning Center on Visual Language and Visual Learning (VL2).

Acknowledgments: The authors also want to express their gratitude to the participants who have joined the study.

Competing Interests: The authors have declared that no competing interests exist.

\section{References}

Andres, M., Michaux, N., \& Pesenti, M. (2012). Common substrate for mental arithmetic and finger representation in the parietal cortex. NeuroImage, 62(3), 1520-1528. https://doi.org/10.1016/j.neuroimage.2012.05.047

Andres, M., \& Pesenti, M. (2015). Finger-based representation of mental arithmetic. In R. Cohen Kadosh \& A. Dowker (Eds.), The Oxford handbook of numerical cognition (pp. 67-88). Oxford University Press.

Andres, M., Seron, X., \& Olivier, E. (2007). Contribution of hand motor circuits to counting. Fournal of Cognitive Neuroscience, 19(4), 563-576. https://doi.org/10.1162/jocn.2007.19.4.563

Badets, A., \& Pesenti, M. (2010). Creating number SEMantics through finger movement perception. Cognition, 115(1), 46-53. https://doi.org/10.1016/j.cognition.2009.11.007

Badets, A., Pesenti, M., \& Olivier, E. (2010). Response-effect compatibility of finger-numeral configurations in arithmetical context. Quarterly fournal of Experimental Psychology, 63(1), 16-22. https://doi.org/10.1080/17470210903134385

Bender, A., \& Beller, S. (2012). Nature and culture of finger counting: Diversity and representational effects of an embodied cognitive tool. Cognition, 124(2), 156-182. https://doi.org/10.1016/j.cognition.2012.05.005

Berteletti, I., \& Booth, J. R. (2015). Perceiving fingers in single-digit arithmetic problems. Frontiers in Psychology, 6, Article 226. https://doi.org/10.3389/fpsyg.2015.00226

Berteletti, I., \& Booth, J. R. (2016). Finger representation and finger-based strategies in the acquisition of number meaning and arithmetic. In D. B. Berch, D. C. Geary, \& K. Mann Koepke (Eds.), Development of mathematical cognition: Vol. 2. Neural substrates and genetic influences. Elsevier. https://doi.org/10.1016/B978-0-12-801871-2.00005-8

Bull, R., Blatto-Vallee, G., \& Fabich, M. (2006). Subitizing, magnitude representation, and magnitude retrieval in deaf and hearing adults. Fournal of Deaf Studies and Deaf Education, 11(3), 289-302. https://doi.org/10.1093/deafed/enj038

Chinello, A., Cattani, V., Bonfiglioli, C., Dehaene, S., \& Piazza, M. (2013). Objects, numbers, fingers, space: Clustering of ventral and dorsal functions in young children and adults. Developmental Science, 16(3), 377-393. https://doi.org/10.1111/desc.12028

Crollen, V., Seron, X., \& Noël, M.-P. (2011). Is finger-counting necessary for the development of arithmetic abilities? Frontiers in Psychology, 2, Article 242. https://doi.org/10.3389/fpsyg.2011.00242

Dehaene, S., Bossini, S., \& Giraux, P. (1993). The mental representation of parity and number magnitude. Journal of Experimental Psychology: General, 122(3), 371-396. https://doi.org/10.1037/0096-3445.122.3.371

Di Luca, S., Granà, A., Semenza, C., Seron, X., \& Pesenti, M. (2006). Finger-digit compatibility in Arabic numeral processing. Quarterly Journal of Experimental Psychology, 59(9), 1648-1663. https://doi.org/10.1080/17470210500256839

Di Luca, S., Lefèvre, N., \& Pesenti, M. (2010). Place and summation coding for canonical and non-canonical finger numeral representations. Cognition, 117(1), 95-100. https://doi.org/10.1016/j.cognition.2010.06.008

Di Luca, S., \& Pesenti, M. (2008). Masked priming effect with canonical finger numeral configurations. Experimental Brain Research, 185(1), 27-39. https://doi.org/10.1007/s00221-007-1132-8 
Di Luca, S., \& Pesenti, M. (2011). Finger numeral representations: More than just another symbolic code. Frontiers in Psychology, 2, Article 272. https://doi.org/10.3389/fpsyg.2011.00272

Domahs, F., Krinzinger, H., \& Willmes, K. (2008). Mind the gap between both hands: Evidence for internal finger-based number representations in children's mental calculation. Cortex, 44(4), 359-367. https://doi.org/10.1016/j.cortex.2007.08.001

Domahs, F., Moeller, K., Huber, S., Willmes, K., \& Nuerk, H.-C. (2010). Embodied numerosity: Implicit hand-based representations influence symbolic number processing across cultures. Cognition, 116(2), 251-266. https://doi.org/10.1016/j.cognition.2010.05.007

Downing, P., \& Kanwisher, N. (2001). A cortical area specialized for visual processing of the human body. fournal of Vision, 1(3), 2470-2473. https://doi.org/10.1167/1.3.341

Emmorey, K., Bosworth, R., \& Kraljic, T. (2009). Visual feedback and self-monitoring of sign language. fournal of Memory and Language, 61(3), 398-411. https://doi.org/10.1016/j.jml.2009.06.001

Fayol, M., Barrouillet, P., \& Marinthe, C. (1998). Predicting arithmetical achievement from neuro-psychological performance: A longitudinal study. Cognition, 68(2), B63-B70. https://doi.org/10.1016/S0010-0277(98)00046-8

Fischer, M. H. (2008). Finger counting habits modulate spatial-numerical associations. Cortex, 44(4), 386-392. https://doi.org/10.1016/j.cortex.2007.08.004

Fischer, M. H., \& Brugger, P. (2011). When digits help digits: Spatial-numerical associations point to finger counting as prime example of embodied cognition. Frontiers in Psychology, 2, Article 260. https://doi.org/10.3389/fpsyg.2011.00260

Gebuis, T., Cohen Kadosh, R., De Haan, E., \& Henik, A. (2009). Automatic quantity processing in 5-year olds and adults. Cognitive Processing, 10(2), 133-142. https://doi.org/10.1007/s10339-008-0219-x

Gerstmann, J. (1940). Syndrome of finger agnosia, disorientation from right and left, agraphia and acalculia. Archives of Neurology \& Psychiatry, 44(2), 398-408. https://doi.org/10.1001/archneurpsyc.1940.02280080158009

Henik, A., \& Tzelgov, J. (1982). Is three greater than five: The relation between physical and semantic size in comparison tasks. Memory \& Cognition, 10(4), 389-395. https://doi.org/10.3758/BF03202431

Holloway, I. D., \& Ansari, D. (2009). Mapping numerical magnitudes onto symbols: The numerical distance effect and individual differences in children's mathematics achievement. fournal of Experimental Child Psychology, 103(1), 17-29.

https://doi.org/10.1016/j.jecp.2008.04.001

Krinzinger, H., Koten, J. W., Horoufchin, H., Kohn, N., Arndt, D., Sahr, K., . . Willmes, K. (2011). The role of finger representations and saccades for number processing: An FMRI study in children. Frontiers in Psychology, 2, Article 373.

https://doi.org/10.3389/fpsyg.2011.00373

Lafay, A., Thevenot, C., Castel, C., \& Fayol, M. (2013). The role of fingers in number processing in young children. Frontiers in Psychology, 4, Article 488. https://doi.org/10.3389/fpsyg.2013.00488

Long, I., Malone, S. A., Tolan, G. A., Burgoyne, K., Heron-Delaney, M., Witteveen, K., \& Hulme, C. (2016). The cognitive foundations of early arithmetic skills: It is counting and number judgment, but not finger gnosis, that count. Fournal of Experimental Child Psychology, 152, 327-334. https://doi.org/10.1016/j.jecp.2016.08.005

Malone, S. A., Burgoyne, K., \& Hulme, C. (2020). Number knowledge and the approximate number system are two critical foundations for early arithmetic development. Journal of Educational Psychology, 112(6), 1167-1182. https://doi.org/10.1037/edu0000426

Newman, S. D. (2016). Does finger sense predict addition performance? Cognitive Processing, 17(2), 139-146. https://doi.org/10.1007/s10339-016-0756-7

Nicoladis, E., Marentette, P., Pika, S., \& Barbosa, P. G. (2018). Young children show little sensitivity to the iconicity in number gestures. Language Learning and Development, 14(4), 297-319. https://doi.org/10.1080/15475441.2018.1444486

Noël, M.-P. (2005). Finger gnosia: A predictor of numerical abilities in children? Child Neuropsychology, 11(5), 413-430. https://doi.org/10.1080/09297040590951550

Padden, C., \& Ramsey, C. (2000). American sign language and reading ability in deaf children. In R. I. Mayberry (Ed.), Language acquisition by eye (pp. 165-189). Lawrence Erlbaum Associates.

Reeve, R., \& Humberstone, J. (2011). Five- to 7-year-olds' finger gnosia and calculation abilities. Frontiers in Psychology, 2 , Article 359. https://doi.org/10.3389/fpsyg.2011.00359

Santens, S., \& Verguts, T. (2011). The size congruity effect: Is bigger always more? Cognition, 118(1), 94-110. https://doi.org/10.1016/j.cognition.2010.10.014

Sato, M., Cattaneo, L., Rizzolatti, G., \& Gallese, V. (2007). Numbers within our hands: Modulation of corticospinal excitability of hand muscles during numerical judgment. Journal of Cognitive Neuroscience, 19(4), 684-693. https://doi.org/10.1162/jocn.2007.19.4.684 
Saxe, R., Jamal, N., \& Powell, L. (2006). My body or yours? The effect of visual perspective on cortical body representations. Cerebral Cortex, 16(2), 178-182. https://doi.org/10.1093/cercor/bhi095

Soylu, F., Lester, F. K., Jr., \& Newman, S. D. (2018). You can count on your fingers: The role of fingers in early mathematical development. fournal of Numerical Cognition, 4(1), 107-135. https://doi.org/10.5964/jnc.v4i1.85

Soylu, F., Raymond, D., Gutierrez, A., \& Newman, S. D. (2018). The differential relationship between finger gnosis, and addition and subtraction: An fMRI study. fournal of Numerical Cognition, 3(3), 694-715. https://doi.org/10.5964/jnc.v3i3.102

Soylu, F., Rivera, B., Anchan, M., \& Shannon, N. (2019). ERP differences in processing canonical and noncanonical finger-numeral configurations. Neuroscience Letters, 705(April), 74-79. https://doi.org/10.1016/j.neulet.2019.04.032

Strauss, A., \& Werner, H. (1938). Deficiency in the finger schema in relation to arithmetic disability (finger agnosia and acalculia). American Journal of Orthopsychiatry, 8(4), 719-725. https://doi.org/10.1111/j.1939-0025.1938.tb05344.x

Taylor-Clarke, M., Kennett, S., \& Haggard, P. (2002). Vision modulates somatosensory cortical processing. Current Biology, 12(3), 233-236. https://doi.org/10.1016/S0960-9822(01)00681-9

Verguts, T., \& Fias, W. (2008). Symbolic and nonsymbolic pathways of number processing. Philosophical Psychology, 21(4), 539-554. https://doi.org/10.1080/09515080802285545

Walsh, V. (2003). A theory of magnitude: Common cortical metrics of time, space and quantity. Trends in Cognitive Sciences, 7(11), 483-488. https://doi.org/10.1016/j.tics.2003.09.002

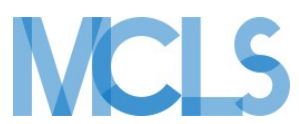

Journal of Numerical Cognition (JNC) is an official journal of the Mathematical Cognition and Learning Society (MCLS).
(P) leibniz-psychology.org

PsychOpen GOLD is a publishing service by Leibniz Institute for Psychology (ZPID), Germany. 